\title{
MASS SPECTROMETRIC STUDY OF DISSOCIATIVE ATTACHMENT IN DIATOMIC MOLECULES. I. CO AND $\mathrm{H}_{2}$
}

\author{
R. LOCHT AND J. MOMIGNY \\ University of Liege (Belgium)
}

\begin{abstract}
A study of the shape of the DA peaks leads to a general method for the determination of the threshold of a DA process. In order to test this method, the negative ions appearing in $\mathrm{CO}$ and $\mathrm{H}_{2}$ have been investigated, using a sector field instrument. An attempt has been made to establish the relative positions of the potential energy curves (using the Morse function) for the electronic states involved in the processes observed.
\end{abstract}

\section{INTRODUCTION}

The aim of this paper is to check with the help of the numerous papers already published on the subject what it is possible to expect from mass spectrometric measurements on the dissociative electron attachment (DA) to diatomic molecules, namely: (i) the way in which the threshold energy values can be determined, (ii) the quantitative interpretation of the DA resonance peak shape as representing the kinetic energy distribution of the negative ion, (iii) the possible deductions concerning the relative position of potential energy curves of $\mathrm{AB}^{-*}$ species decaying into $\mathrm{A}^{-}$and $\mathrm{B}$.

The need for such a check is justified by the subsequent program of measurements that we would like to realize on DA processes and on ion-pair processes in triatomic and polyatomic molecules.

\section{EXPERIMENTAL}

\section{(a) Instrument used and experimental conditions}

Ionization efficiency curves for DA processes were registered by means of a single-focussing $60^{\circ}$ sector mass spectrometer (commercial Atlas CH4). It was verified that the position in energy of the resonance peaks is insensitive to the electron trap current for trap currents below $15 \mu \mathrm{A}$. The electron trap current normally used was fixed at $10 \mu \mathrm{A}$. In all cases it was also verified that the ion intensity was directly proportional to the first power of the pressure in the source region.

The ions were extracted from the ionization region by the penetrating field from the total acceleration electrodes; the extracting electrode was kept at zero potential. The shape of the peaks was not influenced by the value of the total accelerating field. at $2.3 \mathrm{kV}$.

The ion detection was effected using a 17-stage electron multiplier, the first dynode of which was held

\section{(b) Energy distribution of the electron beam}

As will be shown later, the discussion of the experimental results will necessitate the knowledge of the electron-beam energy distribution. The experimental design used in order to measure this distribution is shown in Fig. 1.

The filament $\mathrm{F}$ is heated by a stabilized power supply. The electron beam emitted (E.B.) after passing through the Wehnelt electrode (W) is accelerated by a varying potential difference $V_{A}$ created between $\mathrm{F}$ and the ionization chamber (I.C.). The electron beam is trapped on T where a retarding potential $V_{R}$ is applied. The retarding potential is modulated as shown in Fig. 1 between -15 and +15 volts. The retarding curve obtained is plotted by an $X Y$ recorder. 
Fig. 1. Diagram of the device used for electron energy distribution measurements.

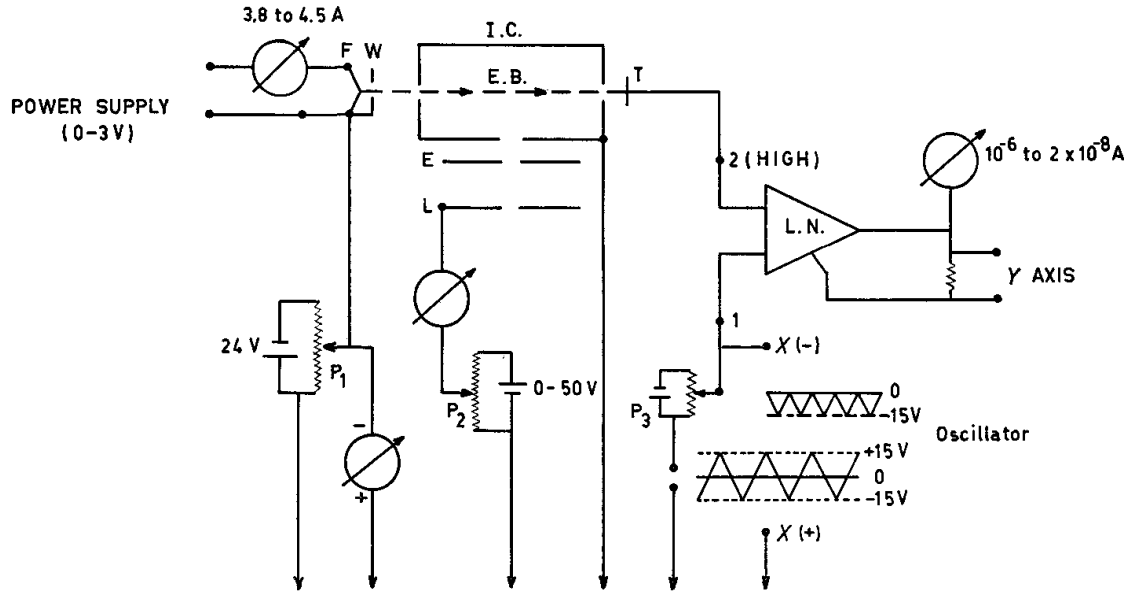

Fig. 2. The electron energy distribution in the ion-source AN4 from the Atlas CH4 mass spectrometer obtained from the first derivative of electron retarding curves.

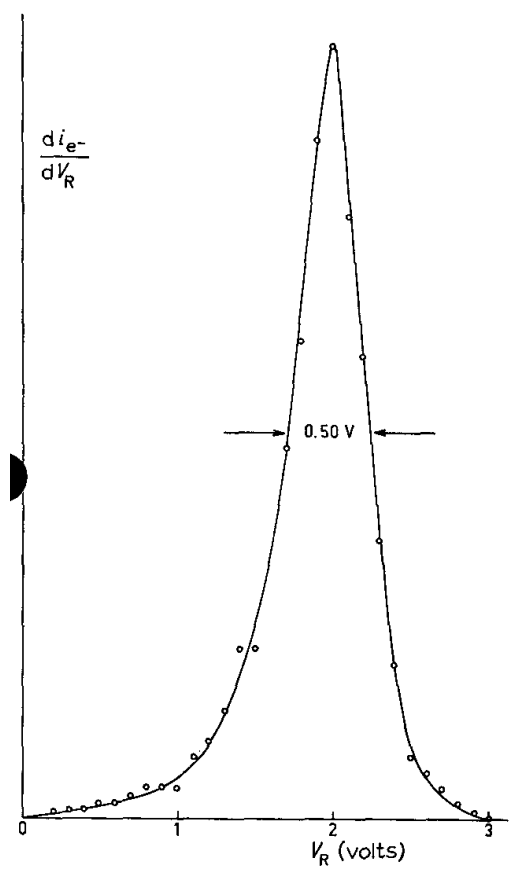

The electronic current intensity against the retarding potential is given by a sigmoidal curve, the first derivative of which is given in Fig. 2. This derivative gives the electron energy distribution of the electron beam. Two important characteristics of the ion source have been verified using this device:

(i) the electron energy distribution is not modified by accelerating voltages of the electrons ranging from 1 to 15 volts;

(ii) with the extraction field, $E$, maintained at zero potential, neither the focussing voltage of the ions applied to $\mathrm{L}$ nor the alteration of the potential of the deflecting electrodes, located after the total accelerating electrode, perturb the electron energy distribution. 


\section{(c) Kinetic energy discrimination of negative ions}

As will be shown, the peak shape gives an image of the KE distributi on of the negative ions. In order to check whether discrimination occurs in the mass spectrometer, the $\mathrm{O}^{-}$peak shape of $\mathrm{O}_{2}$, which is well known as carrying KE from its threshold, has been measured. Its shape has been normalized at the maximum and compared with the total ionization results of Schulz ${ }^{1}$ for which there is no suspicion of KE discrimination. The result of this comparison is given in Fig. 3. The conclusion is that within a range of KE of several volts, we do not observe real discrimination effects on the peak shape.

Fig. 3. The $\mathrm{O}^{-} / \mathrm{O}_{2}$ electron attachment peak normalized to the same peak obtained by Schulz by total ionization. (•) Schulz, (x) this work.

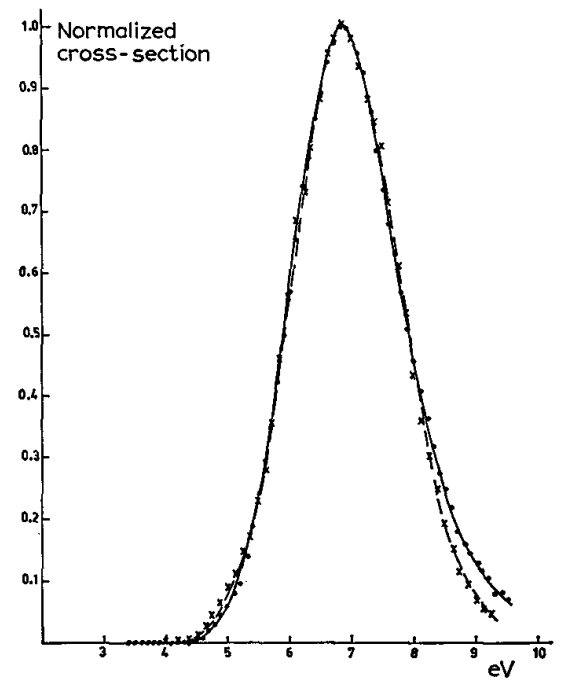

\section{RESULTS FOR CO AND $\mathrm{H}_{2}$}

(a) The only known $\mathrm{DA}$ process in $\mathrm{CO}$ is the appearance of $\mathrm{O}^{-}$. This process has been frequently studied by many authors ${ }^{1-5}$. Some of these used as a calibration of the energy scale the attachment peak of $\mathrm{SF}_{6}$ considered as a zero electron-energy process ${ }^{5}$. Some others used the retarding curve of electrons as a means for this calibration ${ }^{2}$. The attachment curve that we observe is shown in Fig. 4. The shape of this curve and the way in which its threshold should be determined $(a 9.6 \mathrm{eV})$ will be discussed below.

(b) For the $\mathrm{H}_{2}$ molecule the situation is somewhat more complex. Our experimental results are shown in Fig. 5. Two processes are observed: one at $(3.43 \pm 0.15) \mathrm{eV}$ with a maximum at $(4.28 \pm 0.16) \mathrm{eV}$, the width at half height being $0.70 \mathrm{eV}$; the second at $(13.73 \pm 0.08) \mathrm{eV}$ with a maximum at $(14.46 \pm 0.16) \mathrm{eV}$, the width at half height being $0.72 \mathrm{eV}$. Between these peaks, the ionic current due to $\mathrm{H}^{\prime \prime}$ is never zero. However, if we change the potentials of the deflecting plates of the ion source (the normal focussing conditions are such as to maximize the $\mathrm{O}^{-}$ion current from $\mathrm{CO}$ at the maximum of this peak), both processes at $3.43 \mathrm{eV}$ and $13.7 \mathrm{eV}$ disappear and a large peak is found between 7.5 and $14 \mathrm{eV}$, with its maximum at $9 \mathrm{eV}$.

This is consistent with the previous studies of Schulz and Asundi ${ }^{6-8}$ and Rapp et al. ${ }^{9}$ both by massspectrometric and total-ionization measurements. The process appearing between 7.5 and $14 \mathrm{eV}$ is attributed to the mechanism

$$
\mathrm{H}_{2}+\mathrm{e}^{-} \rightarrow\left(\mathrm{H}_{2}^{-}\right)^{*} \rightarrow \mathrm{H}(1 \mathrm{~s})+\mathrm{H}^{-}\left(\mathrm{ls}^{2}\right)
$$

for which a very large excess of $\mathrm{KE}$ is carried away by both $\mathrm{H}$ and $\mathrm{H}^{-}$. This implies that for an ion of small mass $\left(\mathrm{H}^{-}\right)$carrying a large excess of $\mathrm{KE}$, the velocity distribution of the ion is such that a severe discrimination occurs under normal operating conditions of the mass spectrometer. As we have shown, however, with $\mathrm{O}^{-}$from $\mathrm{O}_{2}$, ions with a mass about 10-20 times larger than $\mathrm{H}^{-}$, this effect is not detected. 
Fig. 4. $\mathrm{O}^{-} / \mathrm{CO}$ electron attachment peak (mean of ten runs). The energy scale is corrected.

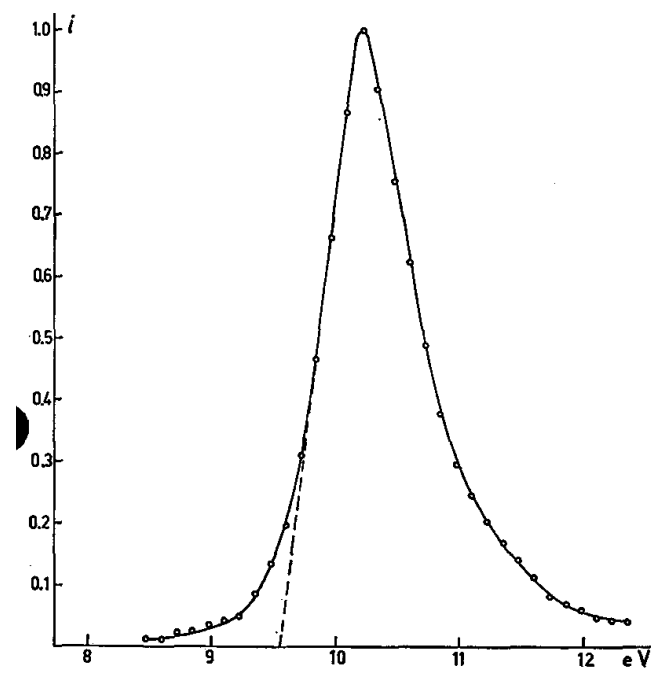

Fig. 5. $\mathrm{H} / \mathrm{H}_{2}$ electron attachment mean peak of six runs. The energy scale is corrected. The cross-section data are taken from refs. 7 and 9: in this work, the ratio $\sigma_{13.73} / \sigma_{3.45}=11.2 \pm 1.6$ (other work: 13.0 or 12.5).

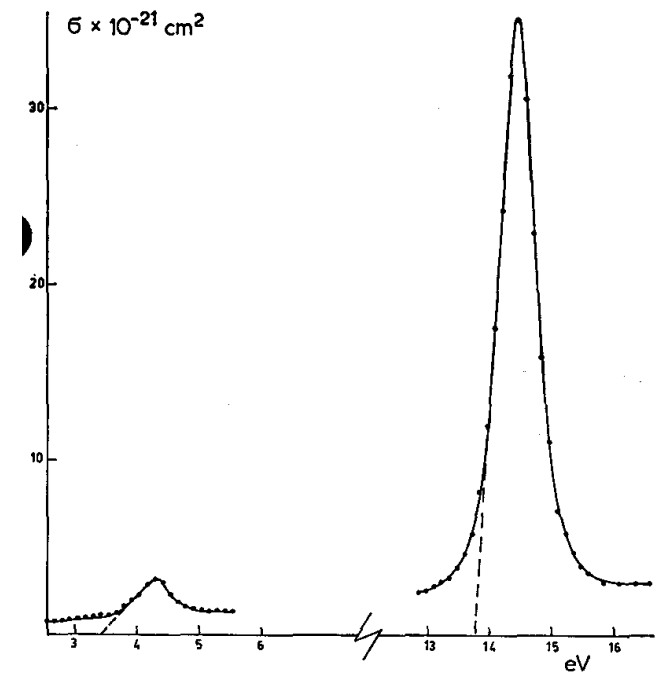

\section{DISCUSSION}

\section{A. Preliminary examination of the theoretical situation}

The following considerations have been discussed many times ${ }^{9-12}$; we believe, however, that it will be helpful to review the situation again in order to give a firm foundation to the discussion of the experimental results.

\section{(a) Peak shape for the $\mathrm{DA}$ process}

The discussion of the peak shape for the DA processes will be presented on the following assumptions: (1) the electron beam used is monoenergetic, and (2) the mass spectrometer does not discriminate against ions carrying excess kinetic energy.

As has been shown above [Experimental point (c), and Results, point (b)], assumption (2) is fulfilled in our experiments but (1) is not: the electron energy distribution of the beam is not negligible, but has however 
been determined. The conclusions of the present discussion will therefore be reconsidered later, through a convolution process taking into account the energy distribution of the electron beam.

As has been shown by some authors ${ }^{12}$ and reviewed by Chantry ${ }^{13}$, the peak shape of the negative ion $\mathrm{A}^{-}$, as expressed by $\sigma(E)$ where $\sigma$ is the cross-section for DA process and $E$ the electron energy, is given by the product of three terms:

$$
\sigma(E) \cong\left(\Gamma_{\overline{\mathrm{a}}} / E V^{\prime}\right)|\psi(E)|^{2} \exp [-\rho(E)]
$$

$\Gamma_{\tilde{\mathbf{a}}}$ is the entrance width to the process, that is to say, the uncertainty in the energy of the intermediate $\left(\mathrm{AB}^{-}\right)^{*}$ state associated with the probability of its decay by autodetachment back to the ground electronic state of the molecule; $V$ is the slope of the intermediate $\left(\mathrm{AB}^{-}\right)^{*}$ potential energy curve; $\psi(E)$ is the initial vibrational wave function written in terms of the electron energy $E$; and $\exp [-\rho(\mathrm{E})]$ is the probability that $\left(\mathrm{AB}^{-}\right)^{*}$ does not decay through autodetachment before dissociating into $\mathrm{A} \sim$ and $\mathrm{B}$.

$$
\text { If we consider as a first approximation that }\left(\Gamma_{\overline{\mathbf{a}}} / E V^{\prime}\right) \exp [-\rho(E)] \text { is constant }
$$

Fig. 6. Potential energy diagram for a diatomic molecule AB: $(X)$ is the ground electronic state, (a), (b) and (c) are hypothetical electronic states of $\left(A B^{-}\right)^{*}$. The Franck-Condon region is limited by dotted lines.

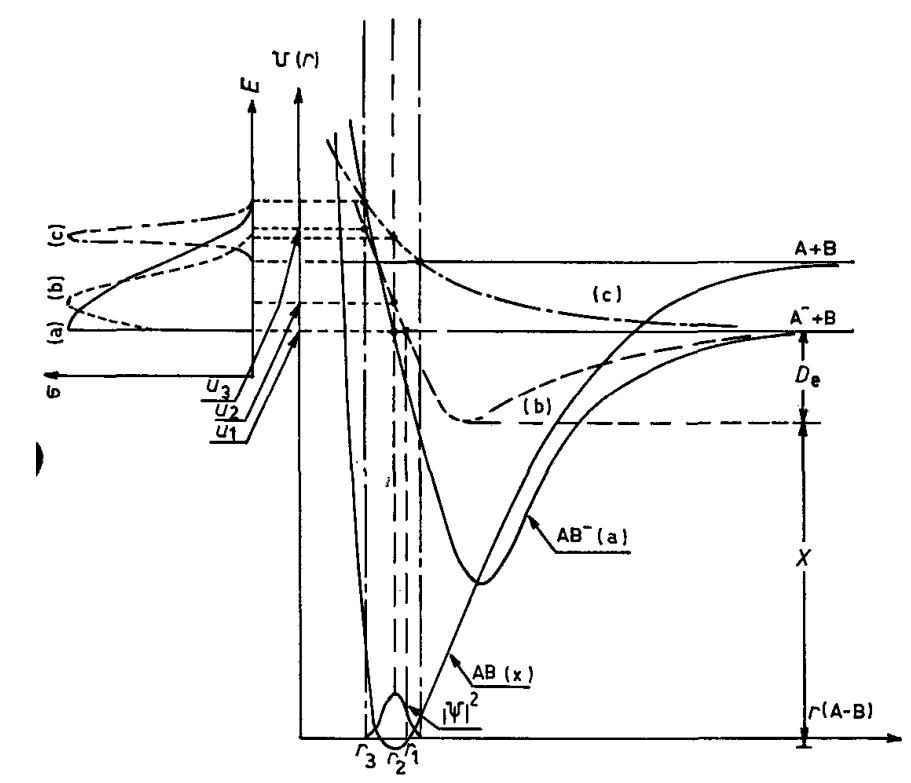

for a given process, $\sigma(E)$ will be approximated by $|\psi(E)|^{2}$, or, better, following the reflection method used in the interpretation of continuous spectra ${ }^{14}$, by the reflection of $|\psi(E)|^{2}$ in that part of the potential energy curve of $\left(\mathrm{AB}^{-}\right)^{*}$ which leads to the appearance of $\mathrm{A}^{-}$. Some situations of this kind have been drawn in Fig. 6 . The peak shapes, corresponding in these cases to the total kinetic energy distributions of $\mathrm{A}$ " and $\mathrm{B}$, are drawn for three processes, (a), (b) and (c).

It may be seen from these distributions, (a) and (b), that if the ions do not have KE at their threshold, the latter will be very sharp; in these cases, the $|\psi(E)|^{2}$ is not completely involved in the DA process.

In the third case, however, where the whole $|\psi(E)|^{2}$ is involved, it will never be possible to assess without independent measurements on the KE distributions of $\mathrm{A}^{-}$and $\mathrm{B}$, whether the fragments formed at the threshold are free from kinetic energy or not.

Before going further into this discussion, it should be kept in mind that in mass spectrometric measurements (as in total ionization measurements), the observed peak shape is not related to the total $\mathrm{KE}$ distribution of $\mathrm{A}^{-}$and $\mathrm{B}$ but with that part of the total KE which is kept only by the $\mathrm{A}^{-}$ion. This was previously 
pointed out by Hagstrum and Tate ${ }^{15}$.

\section{(b) Results of the convolution of the theoretical $\sigma\left(A^{-}\right)$through the electron energy distribution}

Within the limits of the approximation made above in expressing the theoretical peak shape, it is possible to deduce almost qualitatively from the shape of $\sigma\left(\mathrm{A}^{-}\right)$, the most probable potential energy curve $\left(\mathrm{AB}^{-}\right)^{*}$. One possible way to realize this will be outlined below.

Before such an attempt it is however necessary, if the electron beam used is not monoenergetic, to remove from the experimental results the broadening of the $\sigma\left(\mathrm{A}^{-}\right)$shape which is due to the electron energy distribution.

Such deconvolution techniques are well known ${ }^{16}$ but their applicability is limited by the signal-to-noise ratio characterizing the measurements.

We therefore selected a different approach which consists in the convolution by our energy distribution of successive portions of the $\left|\psi_{\mathrm{o}}\right|^{2}$ reflected in the repulsive part of a potential energy curve, treated as a straight line of variable slope.

The result of such a treatment is shown in Fig. 7, where three different types of effective reflected $\left|\psi_{\mathrm{o}}\right|^{2}$ $(f(\mathrm{a}), f(\mathrm{~b})$ and $f(\mathrm{c})$, Fig. 7a) have been convoluted (Fig. 7b) by the electron energy distribution shown in Fig. 2.

Fig. 7. (a) Different parts of the initial vibrational distribution function of $C O$ in the ground vibronic state, (b) Results of convolution of the corresponding models after projection on the potential energy curve; (-) convoluted peak (--) projected model.
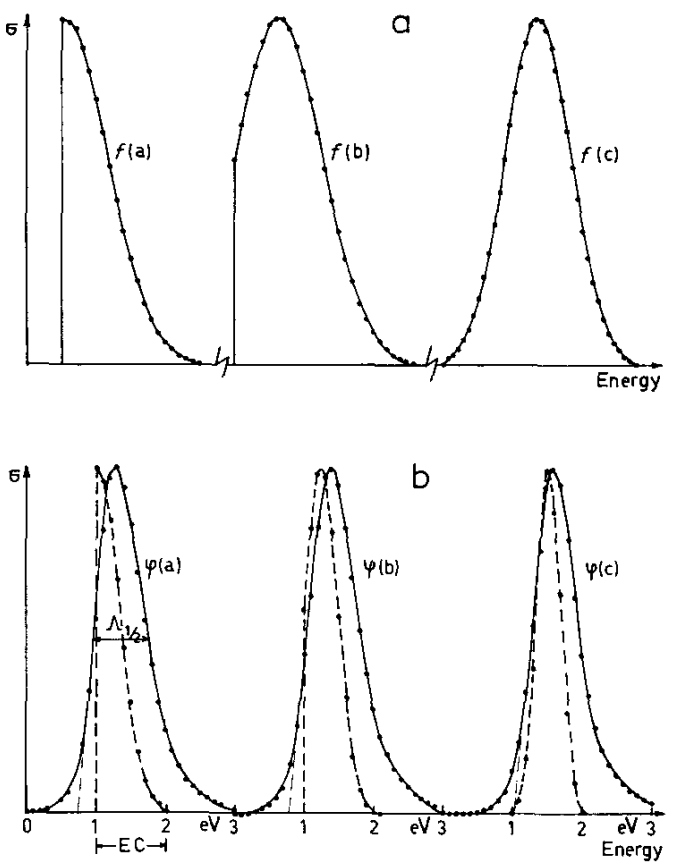

(c) $K E$ of $A^{-}$and its relation with the width at half height of the convoluted models

Using the method described in (b), we have looked for a relation between the width at half height of the convoluted distributions $\Lambda_{1 / 2}$ and the total width at the bottom of the non-convoluted distributions. The relation, which is linear, is shown in Fig. 8 for the three models $f(a), f(b)$ and $f(c)$.

It is therefore possible, after choosing the model for which the convolution best reproduces the experimental curve, to give the value of the KE range carried by $\mathrm{A}^{-}$. 
Fig. 8. The dependence of the width at half height $\left(\Lambda_{1 / 2}\right)$ of the convoluted distributions on the total width (KE) at the bottom of the non-convoluted model. The error lines are drawn to give an idea of the sensitivity of the method used, (a) Linear dependence of $\Lambda_{1 / 2}$. on $\mathrm{KE}$ of $\mathrm{O}^{-} / \mathrm{CO}$, for the three models of Fig. $7 \mathrm{a}$. The slope depends on the portion of $|\psi|^{2}$ involved in the DA process; (b) the same relation for two different molecules, NO and CO, shows that the slope also depends on the specific $|\psi|^{2}$ function for each diatomic species.

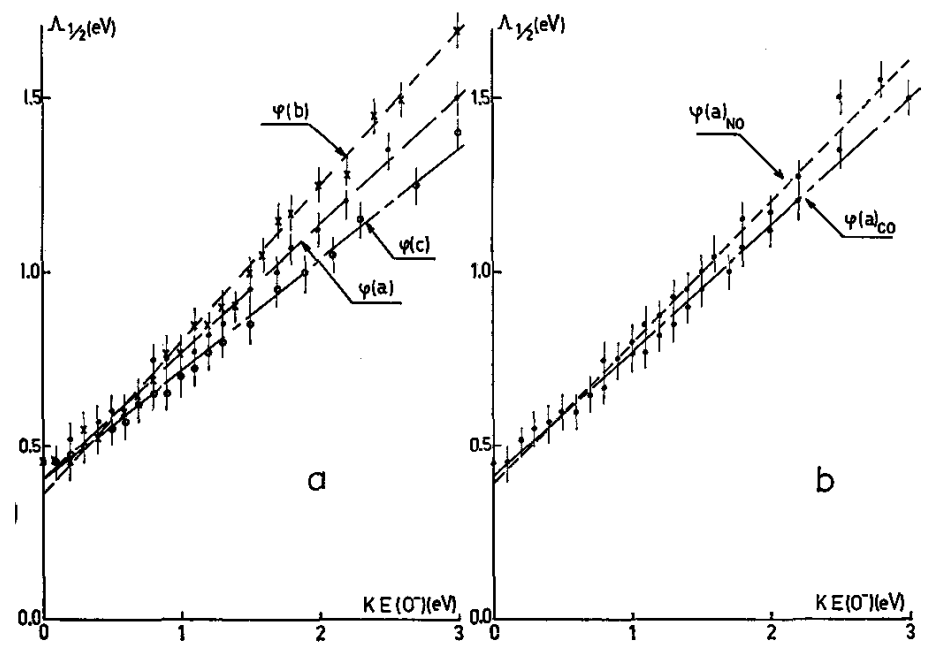

\section{(d) The determination of threshold energies}

We will now consider the way in which the threshold energy for DA processes in diatomic molecules could be most exactly determined within the scope of the approximation made in (A, a).

We shall admit that the intercept on the electron energy axis of the linear extrapolation of the left-hand side of the curve which has the greatest slope, will give the threshold energy.

With this assumption, it is clear from Fig. 7(b) where these extrapolations have been carried out for the three convoluted models $f(\mathrm{a}), f(\mathrm{~b})$ and $f(\mathrm{c})$ that:

(i) in the $f(\mathrm{a})$ and $f(\mathrm{~b})$ cases, the error will be of $0.25 \mathrm{eV}$ by defect, or if we compare two peaks of which the models are $f(\mathrm{a})$ or $f(\mathrm{~b})$, no error will result in the energy difference between the two thresholds;

(ii) in the $f(\mathrm{c})$ case, the error will be negligible, or if we compare two peaks for which the models are of $f(\mathrm{a})$ or $f(\mathrm{~b})$ type with one expressed by the model $f(\mathrm{c})$, an error by excess of about $0.25 \mathrm{eV}$ will be made on the energy difference between the two thresholds.

\section{B. The $\mathrm{O}^{-}$ion in $\mathrm{CO}$}

It is seen from Fig. 9 that the best agreement with the observed peak shape of $\mathrm{O}^{-}$in $\mathrm{CO}$ is given by the convolution of an $f(b)$ type model with a total KE width on $\mathrm{O}^{-}$of $1.1 \mathrm{eV}$.

This implies that at the threshold for the process at $9.6 \mathrm{eV}$

$$
\mathrm{CO}+\mathrm{e}^{-} \rightarrow \mathrm{C}\left({ }^{3} P\right)+\mathrm{O}^{-}\left({ }^{2} P\right)
$$

the $\mathrm{O}^{-}$ions do not carry any KE. This is confirmed by the KE distribution analysis made recently by Chantry ${ }^{13}$.

The $\mathrm{KE}$ carried by $\mathrm{O}^{-}$ions at the maximum of the peak is estimated frorn our model to be approximately $0.3 \mathrm{eV}$, which is in agreement with the results of Chantry ${ }^{13}$. 
We should however keep in mind the discrepancy between the observed peak and the convoluted model: the best explanation for the discrepancy on the high-energy side would be, in part, the existence of an unresolved peak which has a threshold at $10.7 \mathrm{eV}$ and a maximum at $11.1 \mathrm{eV}$. Chantry ${ }^{13}$ resolved this peak by his KE energy analysis technique. This peak is characterized by zero KE and by a threshold at $10.95 \mathrm{eV}$. The process was attributed to the mechanism

$$
\mathrm{CO}+\mathrm{e}^{-} \rightarrow \mathrm{C}\left({ }^{1} D\right)+\mathrm{O}^{-}\left({ }^{2} P\right)
$$

However, the peak which we could obtain by intensity difference between the experimental curve and the convoluted model is made doubtful by the discrepancy observed between the experimental curve and the convoluted model on the low-energy side of the peak; this is to be attributed to the approximations used in the present method.

Fig. 9. (x) Observed electron attachment peak $\mathrm{O}^{-} / \mathrm{CO}$; (o) Convoluted model; (- . -) theoretical cross-section used as model.

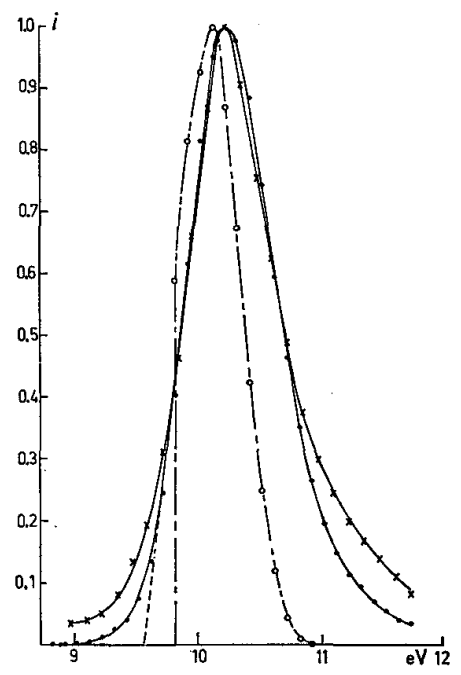

\section{The $\mathrm{H}^{-}$ions in $\mathrm{H}_{2}$}

\section{(a) First process}

It is seen from Fig. 10 (a) that the best agreement with the observed peak shape for the lowest-energy DA process in $\mathrm{H}_{2}$ is given by an $f(\mathrm{~b})$ type model that is very similar to the model used for $\mathrm{O}^{-}$in $\mathrm{CO}$. If we accept that the calculated threshold for the process is $3.72 \mathrm{eV}$, using $\mathrm{D}\left(\mathrm{H}_{2}\right)=4.47 \mathrm{eV}^{14}$ and $\mathrm{EA}(\mathrm{H})=0.75 \mathrm{eV}^{18}$, it is found that the threshold measured by linear extrapolation, with $0 \sim$ from $\mathrm{CO}$ as reference, is at $3.43 \mathrm{eV}$. This result is in excellent agreement with those of Schulz and Asundi ${ }^{6,7}$, which were obtained either with or without using the R.P.D. method.

$$
\mathrm{H}_{2}+\mathrm{e}^{-} \rightarrow H(1 s)^{2}+H(1 s)
$$

The total KE energy width of the peak is found by our method to be $0.9 \mathrm{eV}$.

The discrepancy by defect between the calculated and experimental threshold is very probably related to the factors used in the general expression for $\sigma(E)$ which we chose to neglect as a first approximation. In particular, the survival probability, expressed by $\exp [-\rho(E)]$, could be less than unity for this process. This factor has been successfully taken into account for the calculation of $\sigma(E)$ for $\mathrm{O}^{-}$in $\mathrm{O}_{2}{ }^{17}$. In this case, the survival probability factor shifts the observed peak to lower energies by $1 \mathrm{eV}$ at room temperature. 
Fig. 10. (a) (x) Observed electron attachment peak $\mathrm{H} / \mathrm{H}_{2}$ at $3.45 \mathrm{eV}$; (o) convoluted model; (-.-.) theoretical cross-section used as model; (b) (X) observed electron attachment peak $\mathrm{H}_{1} / \mathrm{H}_{2}$ at $13.72 \mathrm{eV}$; (o) convoluted model; (-.-.) theoretical cross-section used as model.

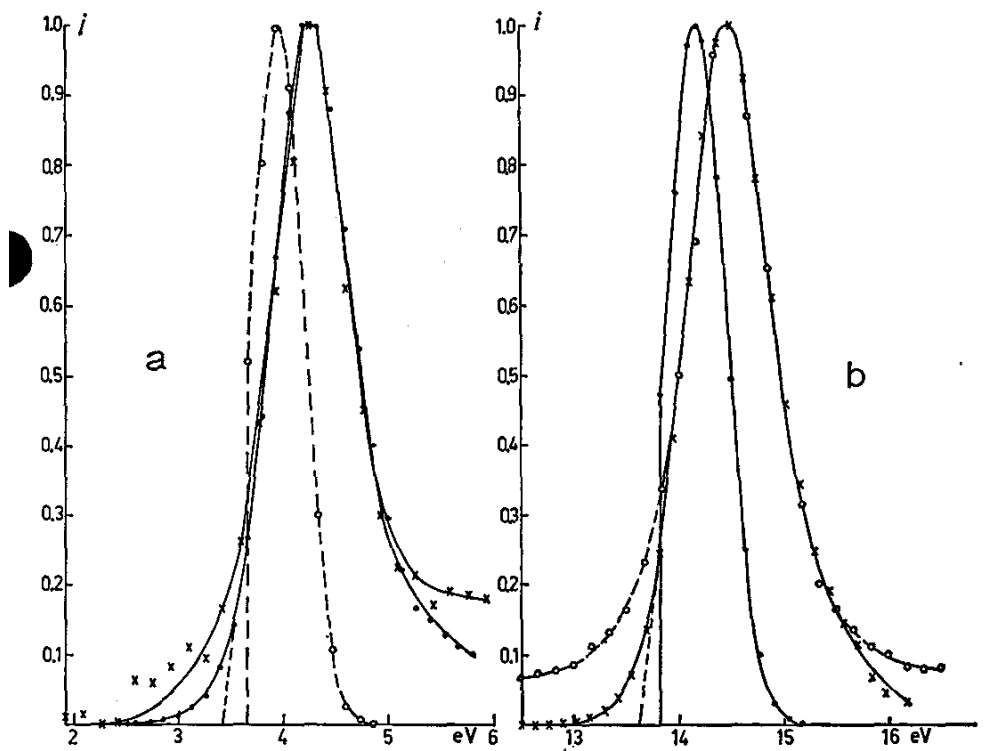

\section{(b) Second process}

The second process is also best fitted by an $f(\mathrm{~b})$ type model as shown in Fig. 10(b). The total width of $\mathrm{KE}$ considered for the $\mathrm{H}^{-}$ion is, from our estimations ${ }^{1} 1 \mathrm{eV}$, which is in agreement with the value given by Schulz ${ }^{8}$. This author estimates the KE to be of the order of $0-0.8 \mathrm{eV}$.

The appearance potential of $\mathrm{H}^{-}$for this process is $13.73 \pm 0.08 \mathrm{eV}$. This is consistent with the mechanism

$$
\mathrm{H}_{2}+\mathrm{e}^{-} \rightarrow\left(\mathrm{H}_{2}^{-}\right)^{*} \rightarrow \mathrm{H}^{-}\left(1 s^{2}\right)+H(2 s)
$$

The difference between the AP for the first process (3.72) eV and the AP for the second (13.73), i.e. 10 $\mathrm{eV}$, is, within the accuracy of our results $( \pm 0.08)$, equal to the excitation energy of the hydrogen atom from its ground state to its first excited (2s) state. The value quoted by Moore ${ }^{19}$ is $10.19 \mathrm{eV}$.

\section{Drawing of the potential energy curves of the $\left(\mathrm{AB}^{-}\right)^{*}$ states}

Together with the first approximations expressed in the preliminary discussion of the results, we have chosen to express the probable potential energy curves of the molecular ground state and of the $\left(\mathrm{AB}^{-}\right)$states by the Morse function. The details of the calculations are given in Appendix 1.

\section{(a) $\mathrm{DA}$ process in $\mathrm{CO}$}

The curve shown in Fig. 11 has been drawn with the following values of the parameters in the Morse function: $\beta=1.27 \times 10^{8} \mathrm{~cm}^{-1}, R_{e}=1.70 \times 10^{-8} \mathrm{~cm}$, and $D_{e}=3.48 \mathrm{eV}$. The dissociation asymptote is given by the threshold value for the process $\mathrm{CO}^{-} \rightarrow \mathrm{C}\left({ }^{3} \mathrm{P}\right)+\mathrm{O}^{-}\left({ }^{2} \mathrm{P}\right)$. The total $\mathrm{KE}$ involved is given by $1.1 \mathrm{eV}^{m}{ }_{c o}{ }^{m}{ }_{o-} \cdot$ In the $\mathrm{MO}$ approximation the ground state of the molecule is described by $\operatorname{KK}_{\cdots}(1 \pi)^{4}(5 \sigma)^{2}(2 \bar{\pi})$.

It follows that the $\mathrm{CO}^{-}$ion in its ground state could be represented as

$$
\mathrm{KK}^{\cdots}(1 \pi)^{4}(5 \sigma)^{2}(2 \pi)^{1} \quad{ }^{2} \Pi
$$

\footnotetext{
${ }^{1}$ The discrepancy between the model and the experimental curve is related (i) to the approximation used, (ii) to the not totally suppressed high- $\mathrm{KE} \mathrm{H}^{-}$ions on the low-energy side of the peak and to a rising ion current on the high-energy side of the peak due to the ion pair $\mathrm{H}_{2} \rightarrow \mathrm{H}^{+}+\mathrm{H}^{-}$
} 
The ${ }^{2} \Pi$ state should be the fundamental state of $\mathrm{CO}^{-}$, which is the compound state responsible for the observations of vibrational excitation in $\mathrm{CO}^{20}$. The curve deduced from the measurements could represent the first excited state of the $\mathrm{CO}^{-}$ion

$$
\mathrm{KK}^{\cdots}(1 \pi)^{4}(5 \sigma)(2 \pi)^{2} \quad{ }^{2} \Sigma \text { or }^{4} \Sigma
$$

and the observed state would then be ${ }^{2} \Sigma$ state of $\mathrm{CO}^{-}$.

Fig. 11. (o) Potential energy curves of the ground vibronic state of $\mathrm{CO} ;(X)$ of the ${ }^{2} \Sigma$ state of $\mathrm{CO}^{-}$; (- - - of the ${ }^{2} \Pi$ state of $\mathrm{CO}^{-}$responsible for the vibrational excitation observed by Schulz ${ }^{20}$.

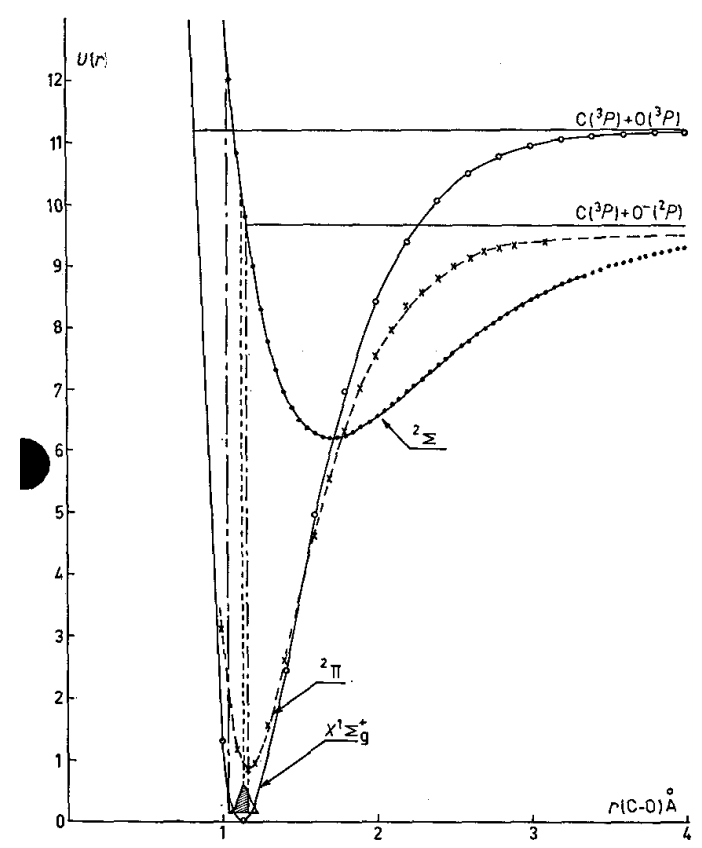

\section{(b) First DA process in $\mathrm{H}_{2}$}

The fundamental state of $\mathrm{H}_{2}^{-}$is described by the electronic configuration $\left(1 s \sigma_{g}\right)^{2}\left(2 p \sigma_{u}\right)^{1}{ }^{2} \sum_{u}{ }^{+}$. Two curves consistent with our results have been drawn in Fig. 12 for the ${ }^{2} \Sigma_{u}{ }^{+}$state of $\mathrm{H}_{2}{ }^{-}$; the lower one is drawn on the basis that the $\mathrm{KE}$ carried by $\mathrm{H}^{-}$is exactly equal to what we deduce from from our model $(0.9 \mathrm{eV})$. A small correction has been made on the linearity approximation of the potential energy curve. The characteristics of ${ }^{2} \Sigma_{u}{ }^{+}$ states of $\mathrm{H}_{2}^{-}$would, in this case, be given by

$$
\beta=1.66 \times 10^{8} \mathrm{~cm}^{-1} R_{e}=1.19 \times 10^{-8} \mathrm{~cm} \quad D_{e}=1.47 \mathrm{eV}
$$

The higher one is drawn on the basis that the $\mathrm{KE}$ carried by $\mathrm{H}^{-}$is naturally uncertain in this case (due to the small cross-section of the DA process giving $\mathrm{H}^{-}$) and could be of about $1 \mathrm{eV}$. In this case the characteristics of the ${ }^{2} \Sigma_{u}^{+}$ state of $\mathrm{H}_{2}{ }^{-}$would be

$$
\beta=2.82 \times 10^{8} \mathrm{~cm}^{-1} \quad R_{e}=1.02 \times 10^{-8} \mathrm{~cm} \quad D_{e}=0.7 \mathrm{eV}
$$

It should be mentioned here that in some theoretical calculations ${ }^{21-23}$ the equilibrium distance $R_{e}$ of the ${ }^{2} \Sigma_{u}{ }^{+}$state of $\mathrm{H}_{2}{ }^{-}$is quoted as being about $0.8 \AA^{2}$ or $1.2 \AA^{23}$.

It follows from these qualitative considerations that the probable region of existence of the ${ }^{2} \Sigma_{u}^{+}$state is given by the shaded zone of Fig. 12. One may see from these results that such curves are able to explain the broad resonances observed by Schulz ${ }^{20}$ about $2.3 \mathrm{eV}$ above the ground vibrational state of $\mathrm{X}^{1} \Sigma_{g}^{+}\left(\mathrm{H}_{2}\right)$. 
Fig. 12. (o) Potential energy curves of the ground vibronic state of $\mathrm{H}_{2} ;(X)$ the ${ }^{2} \Sigma_{u}{ }^{+}$states of $\mathrm{H}_{2}$ : the probable curve lies within the shaded region; $(X)$ the ${ }^{2} \Sigma_{g}{ }^{+}$state leading to $H(2 s)+H(1 s)^{2} ;{ }^{-}{ }^{-}{ }^{2} \Sigma_{g}{ }^{+}$leading to $H(1 s)+H$ $(l s)^{2}$ with KE.

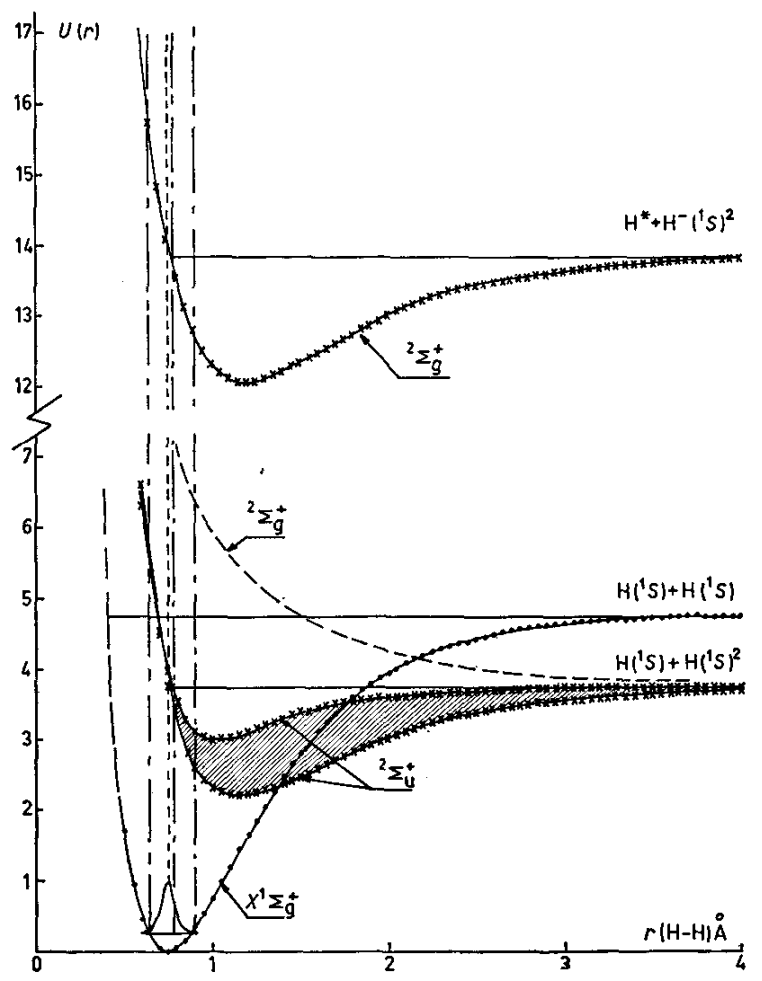

\section{(c) Second $\mathrm{DA}$ process in $\mathrm{H}_{2}$ at $13.7 \mathrm{eV}$}

The theoretical situation above $10 \mathrm{eV}$ has been studied by Eliezer et al. ${ }^{22}$. They describe four states of $\left(\mathrm{H}_{2}^{-}\right)^{*}$ in this region which are the result of adding one electron to the excited parent states of $\mathrm{H}_{2}$. Fig. 12 shows the potential curve which has been deduced from our results using the following characteristics

$$
\beta=1.62 \times 10^{8} \mathrm{~cm}^{-1} \quad R_{e}=1.20 \times 10^{-8} \mathrm{~cm} \quad D=1.73 \mathrm{eV}
$$

This curve compares favorably either with the ${ }^{2} \Sigma_{g}{ }^{+}\left\{\sigma_{g} 1 s \pi_{u} \cdot 2 p \pi_{u} 2 p^{\prime}\right\}$ configuration of Eliezer et al. or with their ${ }^{2} \Sigma_{g}^{+}\left\{\sigma_{g} l s \sigma_{g} 2 s \sigma_{g} 2 s^{\prime}\right\}$ configuration.

\section{(d) The repulsive curve}

The repulsive curve which is responsible for the large peak observed in the total ionization measurements between 7.5 and $14 \mathrm{eV}^{8,9}$ (which is also observed upon alteration of the focussing conditions of the mass spectrometer) is also given in Fig. 12 (dotted curve).

\section{CONCLUSIONS}

This work shows that the study of DA processes in diatomic molecules may be expected to be mass spectrometrically fruitful with the instrument used and that using the assumptions made on the origin of the shape of the cross-section for DA processes as a function of electron energy, thresholds can be determined with accuracy as KE distributions. Another result of this work is that the drawing of potential energy curves for negative molecular ions is only qualitatively possible within the framework of the assumptions we have made.

These results, however, encourage us to use the described technique in further studies of DA processes in diatomic and polyatomic molecules. 


\section{APPENDIX 1}

The quantities $U_{1}\left(r_{1}\right), U_{2}\left(r_{2}\right)$ and $U_{3}\left(r_{3}\right)$ in Fig. 6 have been obtained either by experiment or from our discussion of the experimental results. The molecular characteristics $r_{e}, D_{e}, x$ and $\beta$ for the negative molecular ion have been evaluated from the Morse equation:

$$
\begin{aligned}
& U_{1}=x+D_{\mathrm{e}} \\
& U_{1}=x+D_{\mathrm{e}}\left[1-\exp \left\{-\beta\left(r_{1}-r_{\mathrm{e}}\right)\right\}\right]^{2} \\
& U_{2}=x+D_{\mathrm{e}}\left[1-\exp \left\{-\beta\left(r_{2}-r_{\mathrm{e}}\right)\right\}\right]^{2} \\
& U_{3}=x+D_{\mathrm{e}}\left[1-\exp \left\{-\beta\left(r_{3}-r_{\mathrm{e}}\right)\right\}\right]^{2}
\end{aligned}
$$

Eliminating $x$, one obtains:

$$
-\beta\left(r_{1}-r_{\mathrm{e}}\right)=\ln 2
$$

$\Delta U_{1}+D_{\mathrm{e}}=D_{\mathrm{c}}\left[1-\exp \left\{-\beta\left(r_{2}-r_{\mathrm{e}}\right)\right\}\right]^{2}$

$\Delta U_{2}+D_{\mathrm{e}}=D_{\mathrm{e}}\left[1-\exp \left\{-\beta\left(r_{3}-r_{\mathrm{e}}\right)\right\}\right]^{2}$

where $\Delta U_{1}=U_{2}-U_{1}$ and $\Delta U_{2}=U_{3}-U_{1}$.

Eliminating $r_{\mathrm{e}}$ from eqns. $\left(1^{\prime}\right),\left(2^{\prime}\right)$ and $\left(3^{\prime}\right)$

$$
\begin{array}{r}
\Delta U_{1}+D_{\mathrm{e}}=D_{\mathrm{e}}\left[1-2 \exp \left(\Delta r_{1} \beta\right)\right]^{2} \\
\Delta U_{2}+D_{\mathrm{e}}=D_{\mathrm{c}}\left[1-2 \exp \left(\Delta r_{2} \beta\right)\right]^{2} \\
\text { where } \Delta r_{1}=r_{2}-r_{1} \text { and } \Delta r_{2}=r_{3}-r_{1} .
\end{array}
$$

Combining these equations, one obtains eqn. (3")

$$
\Delta U_{2} / \Delta U_{1}=\exp \left[\left(\Delta r_{2}-\Delta r_{1}\right) \beta\right]\left\{\exp \left(\Delta r_{2} \beta\right)-1\right\} /\left\{\exp \left(\Delta r_{1} \beta\right)-1\right\}
$$

which may be solved graphically with respect to $\beta$. Only one value of $\beta$ satisfies eqn. (3").

In section $\mathrm{A}(\mathrm{b})$ we assume that the repulsive part of the potential energy curve is a straight line. In our calculations, this assumption leads in most cases to large values of $R_{e}$ and $D_{e}$

In the ratio defined in eqn. (3") the quantities $\Delta U_{2}$ and especially $\Delta U_{1}$ are affected by this approximation. Correcting the $\Delta U_{l}$ by $-0.05 m_{A B} / m_{A^{-}}$eV and/or $\Delta U_{2}$ by $+0.05 m_{A B} / m_{A^{-}}$, one obtains the curves discussed above.

\section{ACKNOWLEDGMENT}

We are pleased to thank Professor L. D'Or for his interest in our work. We also thank the Fonds de la Recherche Fondamentale Collective, the Fonds National de la Recherche Scientifique and the University of Liege for the generous help they give to our laboratory.

\section{REFERENCES}

1 G. J. SCHULz, Phys. Rev., 128 (1962) 178.

2 K. KRauss, Dissertation, Hamburg, 1961.

3 D. Rapp And D. D. Briglia, J. Chem. Phys., 43 (1965) 1480.

4 F. H. Dorman, /. Chem. Phys., 44 (1966) 3856

5 P. M. Hierl AND J. L. Franklin, J. Chem. Phys., 47 (1967) 3154.

6 G. J. SchulZ AND R. K. Asundi, Phys. Rev. Letters, 15 (1965) 946.

7 G. J. Schulz And R. K. Asundi, Phys. Rev., 158 (1967) 25.

8 G. J. Schulz, Phys. Rev., 113 (1959) 816.

9 D. Rapp, T. E. Sharp And D. D. Briglia, Phys. Rev. Letters, 14 (1965) 533 
Published in: International Journal of Mass Spectrometry and Ion Physics, (1969), vol. 2, pp.425-440

Status : Postprint (Author's version)

10 J. C. Y. Chen, Phys. Rev., 148 (1966) 66.

11 J. C. Y. ChEn, Phys. Rev., 129 (1963) 202.

12 T. F. O'Malley, Phys. Rev., 150 (1966) 14.

13 P. J. Chantry, Phys. Rev., 172 (1968) 125.

14 G. Herzberg, Spectra of Diatomic Molecules, D. Van Nostrand, New York, 1950, pp. 390

15 H. D. Hagstrum and J. T. TAte, Phys. Rev., 59 (1941) 354.

16 J. D. Morrison, J. Chem. Phys., 39 (1963) 200.

17 T. F. O'Malley, Phys. Rev.. 155 (1967) 59.

18 S. J. Smith AND D. S. Burch, Phys. Rev., 116 (1959) 1125.

19 C E. Moore, Atomic Energy Levels, Circ. Natl. Bur. Std. 467, 1949, Vol. 1.

20 G. J. Schulz, Phys. Rev., 135 (1964) 988.

21 J. N. Bardsley, A. Herzenberg and F. Mandl, Proc. Phys. Soc, (1966) 305.

22 I. Eliezer, H. S. Taylor And J. K. Williams, J. Chem. Phys., 47 (1967) 2165.

23 J. C. Y. Chen And J. L. Peacher, Phys. Rev., 167 (1968) 30. 